\title{
U87MG glioma cells overexpressing IL-17 acclerate early-stage growth in vivo and cause a higher level of CD31 mRNA expression in tumor tissues
}

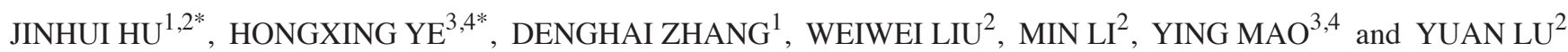 \\ ${ }^{1}$ Department of Lab Medicine, Gongli Hospital, Second Military Medicine University, Pudong New Area, \\ Shanghai 200135; ${ }^{2}$ Department of Lab Medicine, Huashan Hospital, Fudan University, Shanghai 200040; \\ ${ }^{3}$ Department of Neurosurgery, Huashan Hospital, Fudan University, Shanghai 200040; \\ ${ }^{4}$ State Key Laboratory of Medical Neurobiology, Fudan University, Shanghai 200032, P.R. China
}

Received January 11, 2013; Accepted July 30, 2013

DOI: $10.3892 / \mathrm{ol} .2013 .1518$

\begin{abstract}
Immunological alterations have been reported to be involved in glioma, the most common malignant disease of the adult brain. Our recent study identified higher levels of IL-17 in glioma specimens. The present study investigated the role and possible mechanisms of IL-17 in glioma tumorigenesis. Human IL-17 cDNA was cloned and inserted into the eukaryotic pEGFP-N1 expression vector, which was used to transfect the glioma U87MG cell line, resulting in a high level of IL-17 expression in these cells. The cells were then transfected with IL-17 (pEGFP-N1-IL-17-U87MG) or mock (pEGFP-N1-U87MG) vector or left untransfected (U87MG) and subcutaneously inoculated into the right flank of nude mice. The results revealed that the pEGFP-N1-IL-17-U87MG cells grew more rapidly in the early stages $(\mathrm{P}<0.05$, determined on day 32 post-inoculation compared with the other two groups). Quantitative (q)PCR detected higher mouse (m)CD31 mRNA levels in the IL-17-transfected group $(\mathrm{P}<0.01)$ compared with the mock-transfected and untransfected groups. IL-17 transfection altered the mRNA expression of a panel of molecules that are associated with immunity and inflammation in U87MG cells in vitro. An effect of the vector was identified, whereby the mock transfection strongly inhibited cell growth in vivo and dramatically altered the mRNA levels of multiple molecules in the cell culture in vitro compared
\end{abstract}

Correspondence to: Professor Ying Mao, Department of Neurosurgery, Huashan Hospital, Fudan University, 12 Wulmuqui Road, Shanghai 200040, P.R. China

E-mail: yingmaoijc@sina.cn

Professor Yuan Lu, Department of Lab Medicine, Huashan Hospital, Fudan University, 12 Wulmuqui Road, Shanghai 200040, P.R. China E-mail: yuanlu@hsh.stn.sh.cn

${ }^{*}$ Contributed equally

Key words: glioma, IL-17, pEGFP-N1, tumorigenesis, nude mice with the untransfected cells. The present study confirmed that IL-17 overexpression may enhance glioma cell growth in vivo, which may be associated with accelerated angiogenesis. IL-17 overexpression may also alter the cellular mRNA expression of immune-related molecules.

\section{Introduction}

Glioma is the most common malignant disease of the adult brain. The outcome of patients with glioma is poor, mainly due to the diffusion of the tumor into the brain parenchyma (1). Certain immunological dysfunctions have been identified in glioma, including elevated immunosuppressive factors (2-5), reduced total lymphocytes (6), an imbalance in $\mathrm{T}$ helper (Th) subsets (7-14) and the infiltration of immunosuppressive microglia and macrophage cells (15). The immunosuppressive mechanism causes patients to be incapable of eradicating tumor cells and results in the anergy of certain immunotherapies. Therefore, the identification of the role of immune regulatory factors in glioma is significant for obtaining an understanding of the tumorigenesis mechanism and identifying a new therapeutic strategy for this malignant disease.

IL-17 is a main effecter cytokine of Th17 cells and has become a topic of interest following the identification of Th17 in immunology $(16,17)$. IL-17 has been examined in immunology, including autoimmunity, infection, transplantation, allergy and tumors. IL-17 has been shown to promote tumorigenesis via certain mechanisms, including the upregulation of angiogenesis-related molecules, vascular endothelial growth factor (VEGF) and CD31, the activation of the IL-6-STAT3 signaling pathway, the downregulation of IL-12R 32 , thus impairing Th1 function, and the suppression of cytotoxic T lymphocytes (CTLs), causing them to lose their cytotoxic effect via co-operation with CD8 (18-20).

Our recent study identified that IL-17 was expressed at a higher level in glioma tissues compared with trauma tissues (21). Other studies have also demonstrated that IL-17 or Th17 are expressed at higher levels in glioma $(22,23)$. To further explore the role and progress of IL-17 in glioma tumorigenesis, human IL-17 cDNA was cloned and packed 
into the eukaryotic pEGFP-N1 expression vector. The recombinant pEGFP-N1-IL-17 vector was then stably transfected and expressed in the glioma U87MG cell line. The present study investigated the role of IL-17 in promoting glioma tumorigenesis.

\section{Materials and methods}

Recombinant vector and gene amplification. The pEGFP-N1 plasmid was provided by the Institute of Military Medicine Science (Beijing, China) and re-confirmed by sequencing. Peripheral blood $(2 \mathrm{ml})$ was drawn from the peripheral vein from a patient with idiopathic thrombocytopenic purpura (ITP) at the Huashan Hospital (Fudan University, Shanghai, China), according to the Sample Manipulation Guidelines of Huashan Hospital. This study was approved by the ethics committee of Huashan Hospital, Fudan University. Written informed consent was obtained from the patient. Peripheral blood mononuclear cells (PBMCs) were separated by Ficoll centrifugation at $400 \mathrm{x} \mathrm{g}$. The PBMCs were cultured in RPMI-1640 medium supplemented with $100 \mu \mathrm{g} / \mathrm{ml}$ penicillin, $100 \mu \mathrm{g} / \mathrm{ml}$ streptomycin, $2 \mathrm{mM}$ glutamine and $10 \%$ heat-inactivated fetal calf serum (Gibco, Carlsbad, CA, USA). The PBMCs were stimulated for $4 \mathrm{~h}$ with $50 \mathrm{ng} / \mathrm{ml}$ phorbol myristate acetate (PMA; Sigma-Aldrich, St Louis, MO, USA) and $1 \mu \mathrm{M}$ ionomycin in the presence of $10 \mu \mathrm{g} / \mathrm{ml}$ brefeldin A (Alexis Biochemicals, San Diego, CA, USA). mRNA was extracted and cDNA was synthesized using quantitative (q) PCR with primers containing enzymatic digestion sites for BamHI and SalI, according to the manufacturer's instructions. The primers corresponded to NCBI Reference Sequence (NM_002190.2) forward, 5'-CAG TCG ACG ATG ACT CCT GGG AAG ACC TCA TTG-'3 and reverse, 5'-GG TGG ATC CCG GGC CAC ATG GTG GAC AAT CGG-'3. The IL-17 cDNA was packed into a pMD ${ }^{\circledR} 19-\mathrm{T}$ Simple Vector (Takara, Otsu, Japan) to form the pMD19-T-IL-17 vector. Following the sequencing, the recombinant segment of the correct clone was incised by BamHI and SalI (Takara). The recombinant segment was packed into pEGFP-N1, which was incised by the same two restriction endonucleases. The pEGFP-N1-IL-17 clones were sequenced and the correct clones were amplified and identified by restriction enzyme digestion.

Cell line and transfection. The human glioma U87MG cell line was purchased from Cell Bank (Shanghai Life Science Institute, Science Academy of China, Shanghai, China; ATCC no. HTB-14 ${ }^{\mathrm{TM}}$ ). The cells were cultured in Dulbecco's modified Eagle's medium (DMEM) supplemented with $100 \mu \mathrm{g} / \mathrm{ml}$ penicillin, $100 \mu \mathrm{g} / \mathrm{ml}$ streptomycin, $2 \mathrm{mM}$ glutamine and $10 \%$ heat-inactivated fetal calf serum (Gibco). The cells were then cultured at a density of $1 \times 10^{6}$ cells/well in a 6-well plate, and $20 \mu \mathrm{g}$ pEGFP-N1-IL-17 or pEGFP-N1 plasmid were transfected into the U87MG cells using Xfect reagent (Takara), according to the manufacturer's instructions. At $24 \mathrm{~h}$ post-transfection, G418 was added to the culture medium $(200 \mu \mathrm{g} / \mathrm{ml})$. The cells were collected at 10 days post-transfection, when the majority of the cultured cells had died. The remaining cells were diluted to 1 cell/10 $\mu 1$ and $10 \mu 1$ cells was added into the 96 -well plate with G418. The cells were identified by fluorescence and the positive clones were transferred into a 6-well plate. Following amplification for 3 days, the cells were collected for mRNA extraction and qPCR detection (Takara). The following IL-17 primer was used: Forward, 5'-CTG AAC ATC CAT AAC CGG AAT ACC A-'3 and reverse, 5'-AGC GTT GAT GCA GCC CAA G-'3. In order to determine IL-17 secretion, $1 \times 10^{4}$ cells were cultured for 3 days, the culture supernatants were collected and IL-17 was detected using an ELISA kit (R\&D, Minneapolis, MN, USA), according to the manufacturer's instructions.

Mice and xenograft tumor inoculation. Nude mice were purchased and bred at the Animal Laboratory Center, Fudan University. All the mice were handled within a specific pathogen-free facility. All the experimental manipulations were undertaken in accordance with the Guidelines for the Care and Use of Laboratory Animals of Fudan University. This study was approved by the animal ethics committee of Shanghai Medical college, Fudan University. pEGFP-N1-U87MG, pEGFP-N1-IL-17-U87MG and U87MG cells $\left(5 \times 10^{5}\right.$ cells of each type) were inoculated subcutaneously in the right flanks of the nude mice, with 10 mice in each group. The xenograft tumorigenesis effects were observed for the first time at 7 days post-inoculation and monitored once every 3 days. The tumor volumes were measured on days 32 and 35 post-inoculation. The mice were sacrificed on day 39 and the masses and volumes of the xenograft tumors and spleens were measured. Tumor volume (V) was calculated using the formula $V=a b^{2} / 2$ ( $\mathrm{a}$ and $\mathrm{b}$ are the long and short diameters of the tumor, respectively). The RNA of the tumor tissue was extracted and mouse (m)-CXCR2, -CD31, -matrix metalloproteinase 3 (MMP3) and -intercellular adhesion molecule-1 (ICAM-1) were qualified using qPCR. The conditions for the qPCR were $95^{\circ} \mathrm{C}$ for $30 \mathrm{sec}, 95^{\circ} \mathrm{C}$ for $10 \mathrm{sec}$ and $60^{\circ} \mathrm{C}$ for $20 \mathrm{sec}$ for 40 repeats and $95^{\circ} \mathrm{C}-60^{\circ} \mathrm{C}-95^{\circ} \mathrm{C}$ for the melt curve observation. The primers that were used are listed in Table I. Each sample was tested in triplicate and the RNA of the target molecules were normalized to glyceraldehyde 3-phosphate dehydrogenase (GAPDH). The results were calculated as $2^{-\Delta \Delta \mathrm{CT}}$

$q P C R$ determination of $m R N A$ expression in vitro. In addition to using qPCR to detect the four mouse genes being expressed in the tumor tissues, the mRNA of a panel of molecules associated with immune and inflammatory responses in cells cultured in vitro were also detected using this technology. The molecules that were detected were: The chemokines, CXCL1, CXCL5, CXCL8, CXCL10, CXCL11, monocyte chemoattractant protein-1 (MCP-1; CCL2), regulated on activation, normal T cell expressed and secreted (RANTES; CCL5), CCL20, CCR4 and CCR6; the immunology regulation factors, $\beta 2-\mathrm{MG}$, PD-L1, prostaglandin E2 (PGE2), transforming growth factor (TGF)- $\beta$, IL- 6 and STAT3; and the intercellular matrix molecules, MMP3, ICAM-1 and VEGF. The primers for the detection of these genes were human sequence-specific while those that were used for the detection of the genes in the tumor tissues were mouse-specific (with ' $m$ ' prefixing the gene name in this study). The primer sequences are listed in Table I. qPCR was performed using the conditions that were described previously.

Statistical analysis. The statistical analysis was performed using SPSS 11.5 (SPSS, Inc., Chicago, IL, USA). The data 
Table I. Primer sequences.

\begin{tabular}{|c|c|c|c|}
\hline Gene & Forward primer & Reverse primer & Product size, bp \\
\hline GAPDH & TTCGACAGTCAGCCGCATCT & GTGACCAGGCGCCCAATACG & 115 \\
\hline MCP-1 & GGCTGAGACTAACCCAGAAACATC & TGACTGGGGCATTGATTGCAT & 158 \\
\hline RANTES & GCTGCTTTGCCTACATTGCCC & ACTTGGCGGTTCTTTCGGGTG & 118 \\
\hline CXCL1 & GAACGTGAAGTCCCCCGGAC & GCCACCAGTGAGCTTCCTCC & 175 \\
\hline CXCL5 & GCAGCGCTCTCTTGACCACT & ACGCAACGCAGCTCTCTCAA & 169 \\
\hline CXCL8 & AACTTTCAGAGACAGCAGAGCACAC & GCACTCCTTGGCAAAACTGCAC & 173 \\
\hline CXCL10 & TGAGCCTACAGCAGAGGAACCT & TGCTGATGCAGGTACAGCGTAC & 139 \\
\hline CXCL11 & GCCTTGGCTGTGATATTGTGTGC & CTGCTTTTACCCCAGGGCCT & 94 \\
\hline CCL20 & CAGTGCTGCTACTCCACCTCTG & TGCCGTGTGAAGCCCACAATAA & 112 \\
\hline CCR4 & СTTCCTGAGCAAGCCTGGCA & AGGCTCCTCAAGGCAGGTCT & 101 \\
\hline CCR6 & TATTGAGTCACCTCTACTTTCCT & ACTGGAGTCGAAAACATCGCTGA & 147 \\
\hline PD-L1 & TGGTGGTGCCGACTACAAGC & GGGTAGCCCTCAGCCTGACA & 129 \\
\hline STAT3 & AGGAGCATCCTGAAGCTGACCCA & GAGGGTTCAGCACCTTCACCATT & 163 \\
\hline$\beta 2-\mathrm{MG}$ & AGTATGCCTGCCGTGTGAACC & GCGGCATCTTCAAACCTCCAT & 100 \\
\hline PGE2 & TGTTTTGAATGGGCGCCCG & CGGGAACGTTTGCAGACCGT & 173 \\
\hline IL-6 & AAGCCAGAGCTGTGCAGATGA & TGGTTCTGTGCCTGCAGCTT & 136 \\
\hline TGF- $\beta$ & GCCGAGCCCTGGACACCAAC & GCGCCCGGGTTATGCTGGTT & 220 \\
\hline ICAM-1 & AGTCGACGCTGAGCTCCTCT & TGTCTGGGCATTGCCAGGTC & 137 \\
\hline VEGF & GGTGCCCGCTGCTGTCTAAT & CGCCTCGGCTTGTCACATCT & 194 \\
\hline MMP3 & GCTAAGTAAAGCCAGTGGAAATGAA & ACAGGACCACTGTCCTTTCTCC & 199 \\
\hline $\mathrm{mCXCR}^{\mathrm{a}}$ & GTTCAACCAGCCCTGACAGCT & TGGCAGAATAGAGGGCATGCC & 207 \\
\hline $\mathrm{mCD} 31^{\mathrm{a}}$ & GGAAGCCAACAGCCATTACGG & GAGCCTTCCGTTCTCTTGGTGA & 151 \\
\hline mMMP3 $^{\mathrm{a}}$ & GTGTGCTCATCCTACCCATTGC & TAGTGTTGGAGTCCAGCTTCCCT & 211 \\
\hline $\operatorname{mICAM}-1^{\mathrm{a}}$ & TGGCCCTGCAATGGCTTCAA & AGTCTCCAAGCCCAGGCTGA & 196 \\
\hline
\end{tabular}

${ }^{a}$ Gene names that are preceded by ' $\mathrm{m}$ ' indicate that the primer sequence is mouse-specific. Otherwise, the primers are human-specific. GAPDH, glyceraldehyde 3-phosphate dehydrogenase; MCP-1, monocyte chemoattractant protein-1; RANTES, regulated on activation, normal T cell expressed and secreted (CCL5); PGE2, prostaglandin E2; TGF- $\beta$; transforming growth factor- $\beta$; VEGF, vascular endothelial growth factor; MMP, matrix metalloproteinase; ICAM-1, intercellular adhesion molecule-1.

were analyzed by ANOVA and logarithmic transformation was used if necessary. $\mathrm{P}<0.05$ was considered to indicate a statistically significant difference.

\section{Results}

Human IL-17 is successfully expressed in U87MG glioma cells. IL-17 cDNA (NCBI reference sequence, NM_002190.2) was synthesized using RNA that was extracted from PBMCs of an ITP patient. Sequencing and restriction enzyme digestion were used to confirm the successful packages of IL-17 cDNA into the pMD19-T and pEGFP-N1 vectors. The results revealed that the IL-17 cDNA was inserted into the multiple cloning site (MCS) of the pEGFP-N1 vector. The target gene fragment (IL-17 cDNA) was 468bp and the sequence fully corresponded to that in Genbank (Fig. 1).

The cells that were transfected with pEGFP-N1-IL-17 and pEGFP-N1 were selected using $200 \mu \mathrm{g} / \mathrm{ml}$ G418. Following 10 days, the cells were diluted to 1 cell $/ 10 \mu 1$. Subsequent to forming an expansive culture, the cells were identified using a fluorescence microscope and IL-17 mRNA and protein expression was detected by qPCR and an enzyme-linked immunosorbent assay (ELISA). The results revealed that the
U87MG cells that were transfected with pEGFP-N1-IL-17 and pEGFP-N1 exhibited fluorescence, indicating that the vector was expressed successfully in those cells. Notably, the pEGFP-N1-IL-17-U87MG cells demonstrated a significantly higher level of IL-17 mRNA and protein compared with the pEGFP-N1-U87MG and U87MG cells (P<0.001; Fig. 2).

IL-17 overexpression promotes U87MG tumorigenesis in nude mice with elevated CD31 in tumor tissues. pEGFP-N1-IL-17-U87MG, pEGFP-N1-U87MG and U87MG cells $\left(5 \times 10^{5}\right)$ were subcutaneously inoculated into the right flanks of the nude mice. At 7 days post-inoculation, neoplasms became visible and the tumor sizes were monitored every 3 days. At 32 days post-inoculation, the sizes of the neoplasms in the pEGFP-N1-IL-17-U87MG group were larger than those of the pEGFP-N1-U87MG $(\mathrm{P}<0.05)$ and U87MG $(\mathrm{P}<0.05)$ groups. At 35 and 39 days, the tumor volume of the former group remained larger than the latter two groups, but had no statistical significance with the U87MG group, indicating that IL-17 may have accelerated tumor growth at an early stage (Fig. 3).

To explore the possible mechanism underlying the differences in tumor growth among the three groups, the mRNA 
A
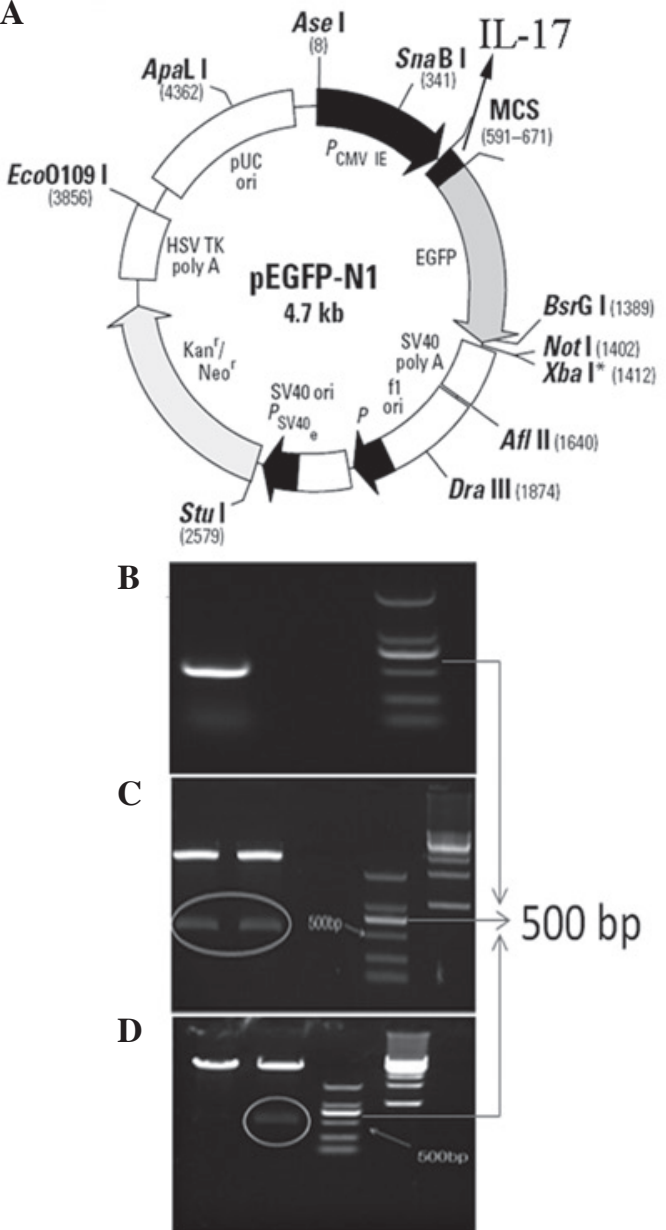

$\mathbf{E}$

Sal

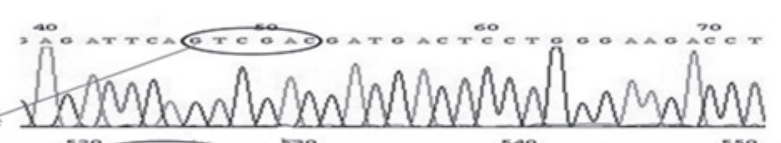

c co\%

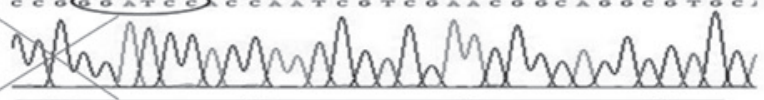
BamH I
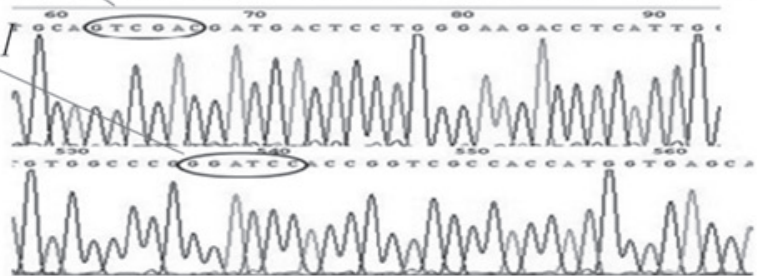

Figure 1. Synthesis of IL-17 cDNA and construction of the pEGFP-N1-IL-17 plasmid. (A) IL-17 cDNA was cloned into the MCS site of pEGFP-N1. (B) PCR for IL-17 cDNA. mRNA was extracted from the PBMCs of an ITP patient and reverse transcribed to cDNA, from which IL-17 was amplified. (C and D) Restriction enzyme digestion of pMD ${ }^{\circledR} 19-\mathrm{T}-\mathrm{IL}-17$ and pEGFP-N1-IL-17 recombinant plasmids showing the IL-17 fragment with $468 \mathrm{bp}$. (E) Sequencing of the pMD19-T-IL-17 and pEGFP-N1-IL-17 recombinant plasmids. SalI and BamHI recognition sequences were identified and the sequence between them fully corresponded to IL-17 from Genbank (accession no. NM_002190.2). MCS, multiple cloning site; PBMC, peripheral blood mononuclear cells; ITP, idiopathic thrombocytopenic purpura.

levels of mCXCR2, mICAM-1, mMMP3 and mCD31 were detected in the tumor tissues. The results revealed a higher mCD31 mRNA level in the pEGFP-N1-IL-17-U87MG group $(\mathrm{P}<0.01)$ compared with the other two groups, while mICAM-1 mRNA was higher in the pEGFP-N1-U87MG group $(\mathrm{P}<0.05)$
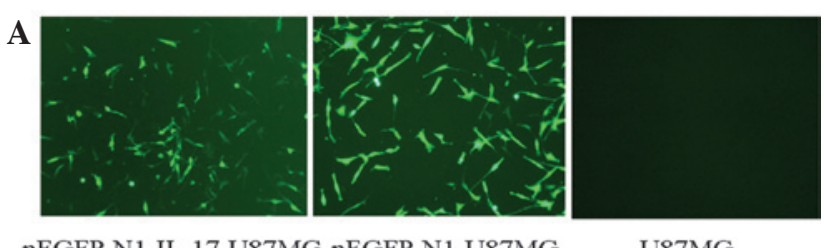

pEGFP-N1-IL-17-U87MG pEGFP-N1-U87MG

U87MG

B

IL-17 mRNA

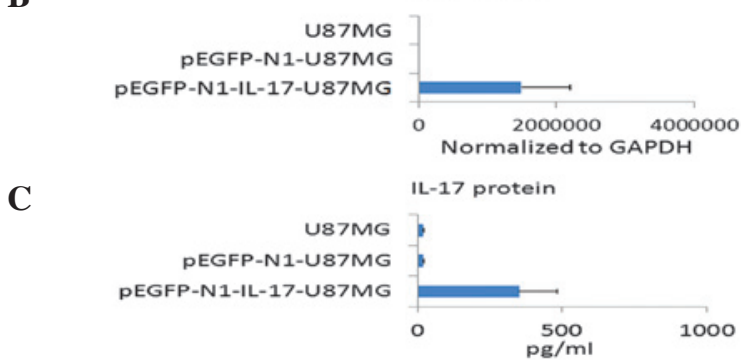

Figure 2. Identification of stably-transfected pEGFP-N1-IL-17-U87MG cells (A) Fluorescence detection of expanded cultured cells from a monoclone. Cells that were transfected with pEGFP-N1-IL-17-U87MG and pEGFP-N1-U87MG exhibited a bright fluorescence, while the untransfected U87MG cells demonstrated no fluorescence. IL-17 (B) mRNA and (C) protein level detection in the three cell lines. The pEGPF-N1-IL-17-U87MG cells presented higher IL-17 mRNA levels in the cell extracts and higher protein levels in the supernatant than the pEGFP-N1-U87MG and U87MG $(\mathrm{P}<0.001)$ cells. When normalized to GAPDH, the IL-17 mRNA levels of the pEGPF-N1-IL-17-U87MG, pEGPF-N1-U87MG and U87MG cells were 1487588 $\pm 708137,1.28 \pm 0.47$ and $1.03 \pm 0.21$, respectively. The IL-17 protein levels in the supernatants of the pEGPF-N1-IL-17-U87MG, pEGPF-N1-U87MG and U87MG cells were $351.6 \pm 130,17.3 \pm 3.6$ and $17.4 \pm 3.5 \mathrm{pg} / \mathrm{ml}$, respectively. GAPDH, glyceraldehyde 3 -phosphate dehydrogenase.
A

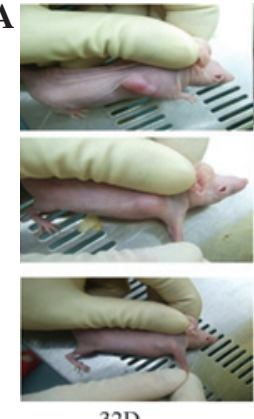

$32 \mathrm{D}$

C 1.5

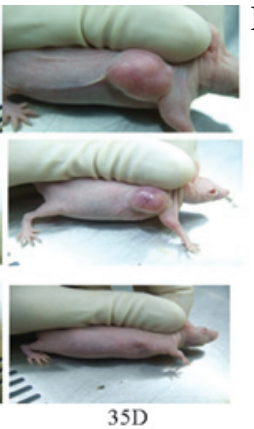

$35 \mathrm{D}$

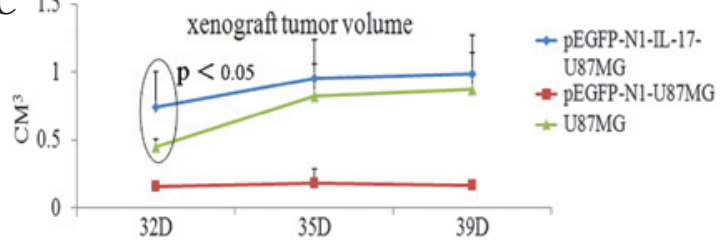

Figure 3. Differential tumorigenesis effect of pEGFP-N1-IL-17-U87MG, pEGFP-N1-U87MG and U87MG cells. (A) At 32 days post-xenograft, the tumor volumes of the pEGFP-N1-IL-17-U87MG mice were higher than those of the U87MG mice $(\mathrm{P}<0.05)$. At days 35 and 39 , the tumor volume remained higher, but lacked statistical significance. Only 6 out of the 10 pEGFP-N1-U87MG mice presented with tumors and the tumor growth rate was slower than of the other two groups at all the time points $(\mathrm{P}<0.01)$. (A) Mice at 32 and 35 days; (B) tumors at 39 days; (C) statistics of the tumor volume at days 32-39.

compared with the other two groups. The levels of mCXCR2 and mMMP3 mRNA were not significantly different among the three groups (Fig. 4). 


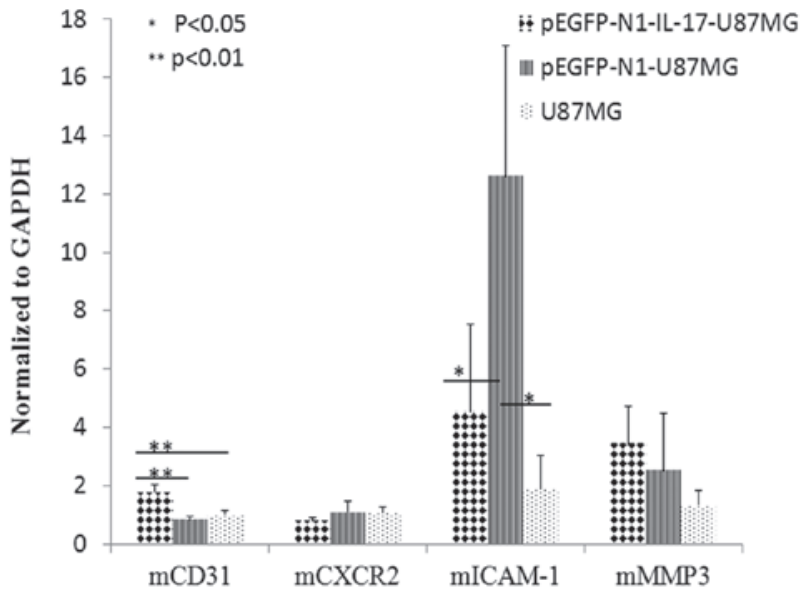

Figure 4. Variation of angiogenesis-related molecules in the xenografted tumors of the pEGFP-N1-IL-17-U87MG, pEGFP-N1-U87MG and U87MG cells. mCD31 mRNA levels were higher in the tumor tissues of the pEGFP-N1-IL-17-U87MG mice than in the other two groups $(\mathrm{P}<0.01)$. mICAM-1 mRNA levels were higher in the tumor tissues of the pEGFP-N1-U87MG mice than in the other groups $(\mathrm{P}<0.05)$. GAPDH, glyceraldehyde 3-phosphate dehydrogenase; mICAM-1, mouse intercellular adhesion molecule-1; mMMP3, mouse matrix metalloproteinase 3.

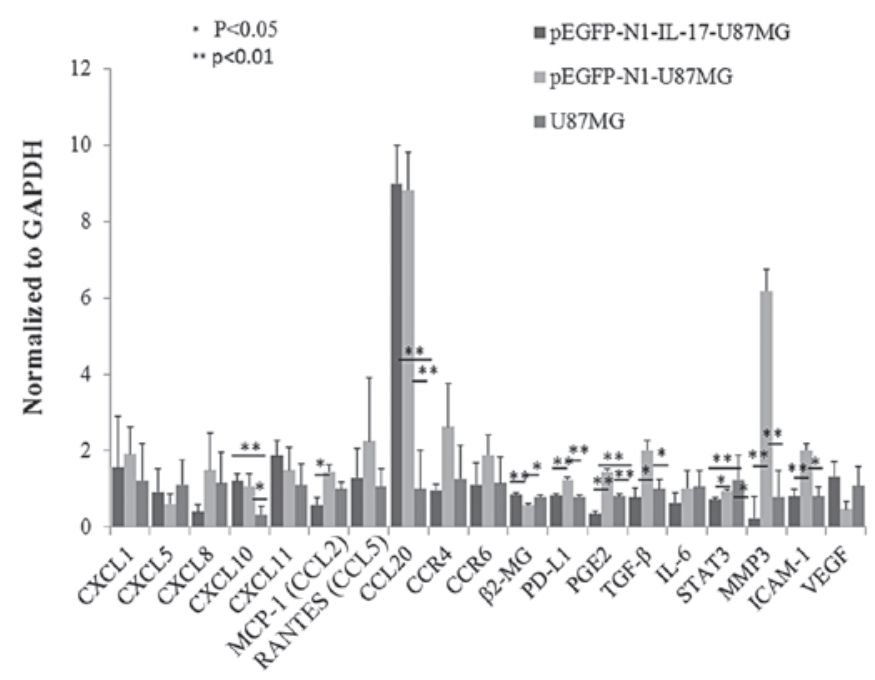

Figure 5. Variation of a panel of immune system- and inflammation-related molecules between pEGFP-N1-IL-17-U87MG, pEGFP-N1-U87MG and U87MG cells. The pEGPF-N1-IL-17-U87MG cells exhibited higher CXCR10 and CCL20 mRNA levels than the U87MG cells $(\mathrm{P}<0.01)$ and higher $\beta$-2MG mRNA levels than the pEGFP-N1-U87MG cells $(\mathrm{P}<0.01)$ The pEGPF-N1-U87MG cells expressed higher CXCL10, TGF- $\beta$, ICAM-1 $(\mathrm{P}<0.05)$, CCL20, PD-L1, PGE2 and MMP3 $(\mathrm{P}<0.01)$ mRNA levels than the U87MG cells and higher MCP-1, TGF- $\beta$, STAT3 $(\mathrm{P}<0.05)$, PD-L1, PGE2, MMP3 and ICAM-1 levels $(\mathrm{P}<0.01)$ than the pEGPF-N1-IL-17-U87MG cells. The U87MG cells expressed higher $\beta$-2MG mRNA levels than the pEGPF-N1-U87MG cells $(\mathrm{P}<0.05)$ and higher STAT3 levels than the pEGPF-N1-IL-17-U87MG cells $(\mathrm{P}<0.01)$. Each group consisted of 6 independent samples, each of which had been tested in triplicate. TGF- $\beta$, transforming growth factor- $\beta$; ICAM-1, intercellular adhesion molecule-1; MMP3, matrix metalloproteinase 3; MCP-1, monocyte chemoattractant protein-1; VEGF, vascular endothelial growth factor; RANTES, regulated on activation, normal $\mathrm{T}$ cell expressed and secreted (CCL5); PGE2, prostaglandin E2.

IL-17 transfection alters the $m R N A$ levels of a panel of immunelinflammation-related molecules in U87MG. To further understand the role of IL-17 in the behavior of U87MG cells, qPCR was used to detect the mRNAs for a panel of proteins that are associated with immune and inflammation responses, including intercellular adhesion, the intercellular matrix and chemokines. The expression of a series of molecules was altered in the pEGFP-N1-IL-17-U87MG, pEGFP-N1U87MG and U87MG cells (Fig. 5).

\section{Discussion}

IL-17, as the main regulatory element of the emerging Th17 subset, has gained considerable interest. Our recent study identified a higher level of IL-17 in glioma tissue (21). The present study identified that the overexpression of IL-17 may accelerate the early-stage growth of U87MG glioma cells in vivo. The expression of Il-17 also altered the mRNA profile of immune/inflammation-related proteins when transfected into a cell culture in vitro.

In the present study, human IL-17 cDNA was inserted into the pEGFP-N1 plasmid and transfected into the glioma U87MG cell line. The success of the procedure was confirmed using gene sequencing, GFP detection and IL-17 mRNA and protein determination. The U87MG, pEGFP-N1-U87MG and pEGFP-N1-IL-17-U87MG cells were inoculated into nude mice. The pEGFP-N1-IL-17-U87MG group demonstrated accelerated tumorigenesis compared with the other two groups when measured at 32 days post-inoculation $(\mathrm{P}<0.05)$. On days 35 and 39 post-inoculation, the implanted tumors of the pEGFP-N1-IL-17-U87MG group were larger than those of the other two groups. However, the difference was not statistically significant. This result indicated that IL-17 was able to accelerate glioma growth, particularly in the early stage of tumorigenesis.

To identify the possible mechanism behind the accelerated tumor growth caused by IL-17 overexpression, mCXCR2, mMMP3, mICAM-1 and mCD31 mRNA expression in the xenografted tumor tissues were analyzed using qPCR. mCD31 expression in the tumor tissues of the pEGFP-N1-IL-17-U87MG group was higher than in the other groups $(\mathrm{P}<0.01)$. This was consistent with the results of a study by Numasaki et al (24). The effect of early-stage tumorigenesis acceleration caused by the overexpression of IL-17 may be associated with the promotion of angiogenesis, which is consistent with the notion that the formation of new blood vessels is vital for the initial growth stage for solid tumors. However, in contrast with the results from the study by Numasaki et al, the present data did not include an elevation in mCXCR2 in the tumor tissues from the IL-17 overexpression group. This difference may have been due to the different tumor types that were used in the two studies. Alternatively, the angiogenesis-promoting effects of IL-17 may also be active through pathways other than CXCR2, as described by a number of studies (25-27). The present study highlights the fact that IL-17 may be a target for interference in tumor angiogenesis.

In addition to detecting the mRNA levels of several molecules in the tumor tissues, the mRNA level for a panel of molecules that are associated with the immune response and inflammation were also analyzed in the transfected cells in order to understand the alterations caused to the behavior of the U87MG cells by IL-17 in vivo. IL-17 was able to increase the levels of CXCL10, CCL20 and $\beta 2-M G$. However, whether these changes are associated with the acceleration of the early-stage 
growth of the U87MG cells in vivo remains to be elucidated. Among the molecules that were studied, $\beta 2-\mathrm{MG}$ was particularly significant, since it was the only one among the observed panel that showed an elevation in the IL-17-transfected cells compared with those that were mock-transfected. $\beta 2-\mathrm{MG}$ was reported to be an early marker in the plasma of hepatocellular carcinoma patients (28). However, the association between $\beta 2-\mathrm{MG}$ and glioma remains elusive and requires further investigation. In the present study, the IL-17-transfected cells contained elevated levels of VEGF mRNA, although they were not increased to a statistically significant level compared with the mock-transfected cells. This elevation of VEGF may be important, since IL-17 tumor-promoting effects are associated with enhanced angiogenesis, as suggested by the present study and others $(24,29,30)$.

Another noteworthy observation in the present study was the effect of the vector; the pEGFP-N1 expression vector was able to dramatically alter the behavior of the U87MG cells in vivo and in vitro. The mock transfection group demonstrated a strong suppression of nude mice xenograft tumorigenesis. On days 32, 35 and 39 post-inoculation, the pEGFP-N1-U87MG group showed a slower rate of tumorigenesis than the other two groups $(\mathrm{P}<0.01)$. In addition, the tumor-inhibiting effects were also revealed by the fact that only six out 10 pEGFP-N1-U87MG mice developed tumors. It is noteworthy that this tumor-inhibitory effect of the vector highlights the tumor-promoting effect of IL-17, since the mock transfection group was a more valued control for the IL-17-transfected group compared with the untransfected group. The in vitro culture also demonstrated the effects of the vector, as there were relatively more molecules showing the alterations in the mRNA levels compared with the IL-17 transfection group. The underlying mechanism for the effect that was observed remains unknown and thus requires further investigation.

In the present study, human IL-17 cDNA was successfully constructed in the glioma U87MG cell line using eukaryotic pEGFP-N1 expression vectors. The pEGFP-N1-IL-17-U87MG cells demonstrated accelerated growth in the early stage subsequent to being inoculated into nude mice, which was accompanied with a higher CD31 expression, indicating that an angiogenesis-promoting action may be involved. IL-17 transfection may also alter the mRNA levels of certain molecules that are associated with the immune response and inflammation. The vector exhibited an effect in the mock transfection group, with suppressed tumor growth and altered mRNA levels of multiple molecules. The mechanism for this phenomenon requires further investigation. The present study revealed that IL-17 was able to enhance glioma growth and change the expression of certain genes.

\section{Acknowledgements}

The authors would like to thank the Central Lab of Huashan Hospital, Fudan University for the instrumental and technical support. This study was supported by grants from the Open Research Fund Program of Shanghai Key Laboratory of Female Reproductive Endocrine-Related Diseases (no. 10dz2220300), the National Distinguish Young Scientists Foundation Grant (no. 81025013) and the Science and Technology Development and Innovation Fund (Pudong New Area, Shanghai, China; no. PKJ2012-Y28).

\section{References}

1. Wen PY and Kesari S: Malignant gliomas. Curr Neurol Neurosci Rep 4: 218-227, 2004.

2. Couldwell WT, Dore-Duffy P, Apuzzo ML and Antel JP: Malignant glioma modulation of immune function: relative contribution of different soluble factors. J Neuroimmunol 33: 89-96, 1991.

3. Nitta T, Hishii M, Sato K and Okumura K: Selective expression of interleukin-10 gene within glioblastoma multiforme. Brain Res 649: 122-128, 1994.

4. Roussel E, Gingras MC, Grimm EA, Bruner JM and Moser RP: Predominance of a type 2 intratumoural immune response in fresh tumour-infiltrating lymphocytes from human gliomas. Clin Exp Immunol 105: 344-352, 1996.

5. Bogdan C and Nathan C: Modulation of macrophage function by transforming growth factor beta, interleukin-4, and interleukin-10. Ann NY Acad Sci 685: 713-739, 1993.

6. Roszman T, Elliott L and Brooks W: Modulation of T-cell function by gliomas. Immunol Today 12: 370-374, 1991.

7. Andaloussi AE, Han Y and Lesniak MS: Progression of intracranial glioma disrupts thymic homeostasis and induces T-cell apoptosis in vivo. Cancer Immunol Immunother 57: 1807-1816, 2008.

8. Heimberger AB, Abou-Ghazal M, Reina-Ortiz C, Yang DS, Sun W, Qiao W et al: Incidence and prognostic impact of FoxP3+ regulatory $\mathrm{T}$ cells in human gliomas. Clin Cancer Res 14: 5166-5172, 2008

9. Brooks WH, Latta RB, Mahaley MS, Roszman TL, Dudka L and Skaggs C: Immunobiology of primary intracranial tumors. Part 5: Correlation of a lymphocyte index and clinical status. J Neurosurg 54: 331-337, 1981.

10. Brooks WH, Netsky MG, Normansell DE and Horwitz DA: Depressed cell-mediated immunity in patients with primary intracranial tumors. Characterization of a humoral immunosuppressive factor. J Exp Med 136: 1631-1647, 1972.

11. Brooks WH, Roszman TL and Rogers AS: Impairment of rosette-forming $\mathrm{T}$ lymphocytes in patients with primary intracranial tumors. Cancer 37: 1869-1873, 1976.

12. Elliott LH, Brooks WH and Roszman TL: Cytokinetic basis for the impaired activation of lymphocytes from patients with primary intracranial tumors. J Immunol 132: 1208-1215, 1984.

13. Roszman TL, Brooks WH, Steele C and Elliott LH: Pokeweed mitogen-induced immunoglobulin secretion by peripheral blood lymphocytes from patients with primary intracranial tumors. Characterization of T helper and B cell function. J Immunol 134: 1545-1550, 1985.

14. Ashkenazi E, Deutsch M, Tirosh R, Weinreb A, Tsukerman A and Brodie C: A selective impairment of the IL-2 system in lymphocytes of patients with glioblastomas: increased level of soluble IL-2R and reduced protein tyrosine phosphorylation. Neuroimmunomodulation 4: 49-56, 1997.

15. Watters JJ, Schartner JM and Badie B: Microglia function in brain tumors. J Neurosci Res 81: 447-455, 2005.

16. Bettelli E, Korn T and Kuchroo VK: Th17: the third member of the effector T cell trilogy. Curr Opin Immunol 19: 652-657, 2007.

17. Harrington LE, Hatton RD, Mangan PR, Turner H, Murphy TL, Murphy KM and Weaver CT: Interleukin 17-producing CD4+ effector $\mathrm{T}$ cells develop via a lineage distinct from the T helper type 1 and 2 lineages. Nat Immunol 6: 1123-1132, 2005.

18. Nam JS, Terabe M, Kang MJ, Chae H, Voong N, Yang YA et al: Transforming growth factor beta subverts the immune system into directly promoting tumor growth through interleukin-17. Cancer Res 68: 3915-3923, 2008.

19. Toh ML, Kawashima M, Zrioual S, Hot A, Miossec P and Miossec P: IL-17 inhibits human Th1 differentiation through IL-12R beta 2 downregulation. Cytokine 48: 226-230, 2009.

20. Wang L, Yi T, Kortylewski M, Pardoll DM, Zeng D and Yu H: IL-17 can promote tumor growth through an IL-6-Stat 3 signaling pathway. J Exp Med 206: 1457-1464, 2009.

21. Hu J, Mao Y, Li M and Lu Y: The profile of Th17 subset in glioma. Int Immunopharmacol 11: 1173-1179, 2011.

22. Wainwright DA, Sengupta S, Han Y, Ulasov IV and Lesniak MS: The presence of IL-17A and T helper 17 cells in experimental mouse brain tumors and human glioma. PLoS One 5: e15390, 2010. 
23. Cantini G, Pisati F, Mastropietro A, Frattini V, Iwakura Y, Finocchiaro G and Pellegatta S: A critical role for regulatory $\mathrm{T}$ cells in driving cytokine profiles of Th17 cells and their modulation of glioma microenvironment. Cancer Immunol Immunother 60: 1739-1750, 2011.

24. Numasaki M, Watanabe M, Suzuki T, Takahashi H, Nakamura A, McAllister F, et al: IL-17 enhances the net angiogenic activity and in vivo growth of human non-small cell lung cancer in SCID mice through promoting CXCR-2-dependent angiogenesis. J Immunol 175: 6177-6189, 2005.

25. Wakita D, Sumida K, Iwakura Y, Nishikawa H, Ohkuri T, Chamoto $\mathrm{K}$, et al: Tumor-infiltrating IL-17-producing gammadelta $\mathrm{T}$ cells support the progression of tumor by promoting angiogenesis. Eur J Immunol 40: 1927-1937, 2010.

26. Moran EM, Connolly M, Gao W, McCormick J, Fearon U and Veale DJ: Interleukin-17A induction of angiogenesis, cell migration, and cytoskeletal rearrangement. Arthritis Rheum 63 $3263-3273,2011$
27. Liu J, Duan Y, Cheng X, Chen X, Xie W, Long H, et al: IL-17 is associated with poor prognosis and promotes angiogenesis via stimulating VEGF production of cancer cells in colorectal carcinoma. Biochem Biophys Res Commun 407: 348-354, 2011.

28. Saito Y, Oba N, Nishinakagawa S, Mizuguchi Y, Kojima T, Nomura K and Nakatsura T: Identification of beta2-microgloblin as a candidate for early diagnosis of imaging-invisible hepatocellular carcinoma in patient with liver cirrhosis. Oncol Rep 23: 1325-1330, 2010.

29. Rapisarda A and Melillo G: Role of the VEGF/VEGFR axis in cancer biology and therapy. Adv Cancer Res 114: 237-267, 2012.

30. Murugaiyan G and Saha B: Protumor vs antitumor functions of IL-17. J Immunol 183: 4169-4175, 2009. 